\title{
Formulation and In vivo Pharmacodynamics studies of Nanostructured Lipid Carriers for Topical Delivery of Bifonazole Running head: Nanostructured Lipid Carriers as vesicles for Topical Delivery of Bifonazole
}

\author{
Aman Deep ${ }^{1}$, Manish Kumar ${ }^{*}$, Rakesh Pahwa², Sumeet Gupta1 ${ }^{1}$, Shailendra Bhatt, \\ Beena Kumari ${ }^{3}$, Prabhat Kumar Upadhyay ${ }^{5}$, A. Pandurangan ${ }^{1}$ \\ 1 M.M College of Pharmacy, Maharishi Markandeshwar (Deemed to be University), Mullana, Ambala, Haryana, India \\ 2 Institute of Pharmaceutical Sciences, Kurukshetra University, Kurukshetra-136119, India \\ 3 Department of Pharmaceutical Sciences, Indira Gandhi University, Meerpur, Rewari, Haryana, India, Pharmaceutics, \\ Rewari, India \\ 4 Institute of Pharmaceutical Research, GLA University, Mathura, Uttar Pradesh,, Pharmaceutical Chemistry, Mathura, \\ India
}

\begin{abstract}
The main objective of the present study was to developed and evaluated the Bifonazole (BFZ) loaded Nanostructured lipid carriers (NLCs) for topical delivery of BFZ. BFZ-NLCs were deve26ted for Particle size, EE, DL and drug release profiles. The invitro release studies show better drug release over $24 \mathrm{~h}$ as compared to the marketed formulation. Ex-vivo skin permeation and Pharmacodynamic studies indicated that NLCs get effectively reduced the fungal infection. In-vitro antifungal activity study shows that the BFZ-NLCs were more effective in inhibiting the growth of Candida Albicans. Therefore, the study concludes that NLCs showed a continuous release profile and has the prospective for treatment of topical fungal infections.
\end{abstract}

Corresponding author:

M.M College of Pharmacy, Maharishi Markandeshwar (Deemed to be University), Mullana, Ambala, Haryana, India

Telephone number: 07017548594

E-mail address: manish_singh17@rediffmail.com

ORCID

Deep Aman 0000-0002-1103-4228,

Manish Kumar 0000-0001-9405-3311,

Rakesh Pahwa 0000-0002-4399-328X,

Beena Kumari 0000-0002-6333-5081,

Prabhat Upadhyay 0000-0001-6825-7126,

Shailendra Bhatt 0000-0001-9405-3311,

Pandurangan A 0000-0002-0379-9853

(Received 09 December 2019, accepted 14 March 2020) 
Keywords: Bifonazole, Nanostructured lipid carriers, Topical delivery, Anti-fungal infection, High pressure homogenization

\section{INTRODUCTION}

In the recent scenario, fungal infections are widely spread all over the world. Billions of people are treated every year with either topical or severe systemic fungal infections ${ }^{1}$. Although, antifungal drugs are effective in treating fungal infections but they are associated with severe toxicities like liver damage or they may affect estrogen levels or may cause allergic reactions. For example, the antifungals with azole groups are known to have caused anaphylaxis.

$\mathrm{BFZ}$ is an azole derivative and active against the fungal infections such as moulds, yeasts, dermatophytes and Gram-positive bacteria. BFZ is mainly preferred for the treatment of topical infections as like tinea pedis, cutaneous candidiasis. And it is virtually insoluble in water with 1-2 h of half-life ${ }^{2,3}$.

Mycelium fungi can deeply penetrate the skin layers and causes the fungal infection. To overcome this problem, improvement in the activity of the active agent for the antifungal treatment is required ${ }^{4}$. Thus, the NLCs based gel was developed for the deeper penetration and retention of the active agents throughout the skin layers. Hence, the purpose of the recent study was to develop the NLCs based gel of BFZ for the fungal infection ultimately increases the effectiveness of the drug.

Nowadays lipids used in the novel drug delivery system. Because lipids plays a important role in improving the bioavailability and enhances the solubility of the lipophilic drugs ${ }^{5}$. NLCs are the advanced form of the nanoparticles. Hence, they overcome the limitations over the conventional formulations and SLNs ${ }^{6,7}$. Previously, SLNs concerned lot of consideration as a drug delivery system ${ }^{8}$. Because they help to improves the bioavailability and increases the solubility of poorly water soluble drugs ${ }^{9}$. However, depending on the drug, a variety of possible problems can occur, such as insufficient drug loading and drug leaking during storage. Later on, NLCs are developed and designed by mixing the solid lipid with the liquid lipid, which leads to exceptional nanostructures with enhanced properties for remedial loading, modification of the drug release profile and stability ${ }^{10,11}$.

NLCs are the advanced form of the nanoparticles and have the reason that they formed a less ordered lipid matrix with many imperfections has the capacity to integrate big quantities of drugs ${ }^{12}$. The primary site of action of Terbinafine is the stratum corneum in fungal infections residing superficially on the skin layers ${ }^{13,14}$.

In this context, topical NLC may prove to be a potential option for increasing the concentration of drug by controlled targeting up to deeper skin layers. SLNs 
involve the incorporation of the solid lipid whereas NLCs involve the drug entrapment into the solid and liquid lipid mix which may contribute to make it a sustained release formulation and thereby overcoming the limitation of SLNs. Topical NLC formulation aims to reach the target site with required concentration to achieve its therapeutic action with minimal adverse effects ${ }^{15,16}$.

Therefore NLCs, the newer generation of SLNs were chosen to overcome these limitations and provide better therapeutic prospects. As a result, the objective of the present study was to prepare and optimize. BFZ loaded NLC for topical administration and prepared formulations for in vitro release, ex vivo permeation and in vivo (Pharmacodynamics) studies which may be found to be more effective than the SLNs developed before.

\section{METHODOLOGY}

\section{Materials}

BFZ was received as a gift sample from Vital Laboratories Private Limited (Gujarat, India). Stearic acid and Castor oil was obtained from Central Drug House Ltd. Vardaan House, Daryaganj, New Delhi (India). Tween8o was obtained from Central Drug House Ltd. Vardaan House, Daryagan (New Delhi, India). Sodium hydroxide pellets, HCL (Concentrated), Methanol AR were obtained from Qualikems Fine Chemicals (Mumbai, India). All reagents and solvents used were of systematic rank.

\section{Experimental design of BFZ loaded NLCs}

A $3^{2}$ full factorial was applied to design the experiments. Polymer ratio and Castor oil were used as independent variables, whereas particle size, percentage entrapment efficiency and percentage Drug loading were kept as dependent variables. Formulations F1 to F9 were prepared using three different levels of lipid ratio and surfactant and the response parameters were evaluated (Table 1).

\section{Selection of lipids:}

The semi quantitative method was used to check the solubility of BFZ in the various solid lipids ${ }^{17}$.the predetermined amount of the drug was precisely weighed in the series of test tubes. Different lipids were included increasing sum to particular test tubes and warmed till medication is absolutely solubilized. The temperature of test cylinders was kept up $10^{\circ} \mathrm{C}$ over the softening purpose of lipid utilized and shaken irregularly to break down the medication. Test tubes were watched outwardly for any medication buildup. The measure of lipid required for solubilizing fixed measure of medication was resolved ${ }^{18,19}$. 


\section{Selection of solid lipids}

The semi quantitative method was used to check the solubility of BFZ in the various solid lipids ${ }^{20}$. The predetermined amount of the drug was precisely weighed in the series of test tubes. Different lipids were included increasing sum to particular test tubes and warmed till medication is absolutely solubilized. The temperature of test cylinders was kept up $10^{\circ} \mathrm{C}$ over the softening purpose of lipid utilized and shaken irregularly to break down the medication. Test tubes were watched outwardly for any medication buildup. The measure of lipid required for solubilizing fixed measure of medication was resolved ${ }^{21}$.

\section{Selection of liquid lipid (oils) and surfactants}

The solubility of BFZ in various liquid lipids (Castor oil, Oleic acid, Isopropyl myristate, Cremophore EL) and surfactants (Tween 20, Tween 80, Span 80 and Pluronic F 127) was determined by adding excess amounts of drug in $3 \mathrm{ml}$ of oils in small vials. The vials were tightly stoppered and were continuously stirred to reach equilibrium for $72 \mathrm{~h}$ at $25^{\circ} \mathrm{C}$ in a mechanical shaker. After that, High Speed Centrifuge (3K30, SIGMA, Germany) were used to centrifuge the mixtures at $15000 \mathrm{rpm}$ for $45 \mathrm{~min}$ at $37^{\circ} \mathrm{C}^{22}$. The upper layer was separated out and solubility was determined by UV Spectrophotometer at $254 \mathrm{~nm}$. The solubility studies were done in triplicate and results reported as $\pm \mathrm{SD}$.

\section{Selection of binary lipid phase}

The solid and liquid lipid with the best-solubilizing potential for BFZ were mixed in different ratios viz., 95:5, 90:10, 85:15, 80:20, 70:30, and 60:40 in array to found the miscibility of the two lipids. Lipid mixtures were agitated at $200 \mathrm{rpm}$ for $1 \mathrm{~h}$ at $85^{\circ} \mathrm{C}$ using a magnetic stirrer (Remi instruments Ltd., Mumbai, India). Smearing a cooled sample of the solid mixture onto a filter paper was used to find out the miscibility between the two components, the visual observation is used to determine the presence of any liquid oil droplets on the filter paper. The binary mixture who shows the melting point above $40^{\circ} \mathrm{C}$ which did not expose the presence of oil droplets on the filter paper was selected for the development of BFZ - loaded NLCs ${ }^{23}$.

\section{Preparation of NLCs}

The weighed amount of drug was added to the lipid phase which was heated at $10-15^{\circ} \mathrm{C}$ above the melting point of solid lipid and simultaneously, aqueous surfactant solution was heated at the same temperature $\left(85^{\circ} \mathrm{C}\right)$. Then the lipid mixture was poured in the hot aqueous surfactant solution using a magnetic stirrer (Remi instruments Ltd., Mumbai, India) at 12,000 rpm for $30 \mathrm{~min}$, to prepare the primary emulsion ${ }^{24}$. 
This primary emulsion was converted to the NLC system using high pressure homogenizer (Stansted Fluid Power Ltd., Harlow, UK) at 15000 PSI. The obtained NLC dispersion was cooled down to room temperature. The NLC dispersion was lyophilized for long term stability. Mannitol ( $5 \% \mathrm{w} / \mathrm{v})$ was added as cryoprotectant. The samples were frozen at $-78^{\circ} \mathrm{C}$ for $10 \mathrm{~h}$ followed by lyophilization for 36 h. The lyophilized formulation was reconstituted with phosphate buffer $\mathrm{pH} 6.8$ as per the requirements for later experiments.

\section{Evaluation and Characterization of BFZ loaded NLCs}

\section{Particle size analysis}

The particle size analysis of NLC formulations was done by photon correlation spectroscopy (PCS) with a Zetasizer (Malvern Instruments, Worcestershire, UK). The PCS provides the mean particle size (z-average).

\section{Entrapment Efficiency (EE) and Drug-loading capacity (DL)}

For EE and DL, the drug-loaded NLC dispersion was uniformly mixed by gentle shaking. $1.0 \mathrm{ml}$ of this dispersion was diluted with $9.0 \mathrm{ml}$ methanol, centrifuged using High-Speed Refrigerated Centrifuge ( $3 \mathrm{~K}_{3}$ o, SIGMA, Germany) for $45 \mathrm{~min}$ at $15,000 \mathrm{rpm}$ and then filtered using Millipore membrane $(0.2 \mu \mathrm{L})$. The analytical method employed was as per the method reported ${ }^{25}$. Hence, UV absorption spectra of stock solution ( $10 \mu \mathrm{g} / \mathrm{mL}$ ) were scanned for absorbance in the region of 400-200 $\mathrm{nm}$ at $254 \mathrm{~nm}$. Serial dilutions of standard solutions were prepared and absorbance was recorded at $254 \mathrm{~nm}$. The calibration curve was prepared and the method was validated. The filtrate was collected and appropriately diluted with methanol and measured spectrophotometrically (Shimadzu, model UV-1601, Kyoto, Japan) at $\kappa_{\max }$ of $254 \mathrm{~nm}$. The percent entrapment efficiency (EE \%) was calculated using the following equation ${ }^{26}$.

$$
\begin{aligned}
& \% \mathrm{EE}=\frac{\mathrm{W}(\text { Total }) \times \mathrm{W}(\text { Free })}{\mathrm{W}(\text { Total })} \times 100 \\
& \% \mathrm{DL}=\frac{\mathrm{W}(\text { Total }) \times \mathrm{W}(\text { Free })}{\mathrm{W}(\text { Lipid })} \times 100
\end{aligned}
$$

$\mathrm{W}_{\text {total }}=$ the weight of drug

$\mathrm{W}_{\text {free }}=$ weight of drug in supernatant

$\mathrm{W}_{\text {lipids }}=$ weight of lipid 


\section{Evaluation of BFZ loaded NLC}

\section{In vitro drug release study}

In vitro drug release studies of NLCs were performed using dialysis bag technique. The activation of dialysis membrane was carried out. The experiments were carried out under sink conditions. $10 \mathrm{mg}$ of each formulation i.e., (F1-F11) was loaded into a cellulose membrane dialysis bag immersed in $200 \mathrm{~mL}$ of $\mathrm{pH} 6.8$ phosphate buffer containing $0.8 \%$ tween 80 solution magnetically stirred at $32^{\circ} \mathrm{C}$ at $\mathrm{pH}$ 6.8. Samples were taken at predetermined intervals from the receiver solution, replaced with equal volumes of fresh solvent, and spectrometrically assayed for drug concentration at $K_{\max } 254 \mathrm{~nm}$. The correction for the cumulative dilution was calculated. The release studies were performed in triplicate ${ }^{27,28}$.

\section{Differential scanning Calorimetry (DSC) study}

Drug lipid interaction in NLCs formulations and crystallinity of drug was analyzed by performing DSC analysis. Samples were analyzed using SII Nanotechnology EXSTAR DSC 6220 in scanning range of $30-300^{\circ} \mathrm{C}$ at a heating rate of $10^{\circ} \mathrm{C} / \mathrm{min}$. Plain drug, lipid, Drug-lipid physical mixture and NLCs formulation DSC scans were recorded and compared.

\section{Transmission electron microscopy (TEM)}

TEM studies were determined for the NLCs using TEM (TECNAI-G2, $200 \mathrm{kV}$, HR-TEM, FEI, The Netherlands). A drop of NLC was placed on a paraffin sheet and carbon coated grid was put on sample and left for 1 min to allow NLCs to adhere on the carbon substrate. The remaining NLC was removed by adsorbing the drop with the corner of a piece of filter paper. Then the grid was placed on the drop of phosphotungstate (1\%) for $10 \mathrm{~s}$. The remaining solution was removed by absorbing the liquid with a piece of filter paper and samples were air dried and examined by TEM.

\section{Preparation of BFZ loaded NLCs Based Gels}

The gels were prepared by dispersing $1 \% \mathrm{w} / \mathrm{w}$ Carbopol 940 in the selected NLCs formulations and subsequently neutralizing the Carbopol_dispersion using triethanolamine (TEA). The final concentrations of BFZ in the NLCs gels were maintained at $0.5,1$ and $1.5 \% \mathrm{w} / \mathrm{w}$ and were coded as G1, G2 and G3 respectively.

\section{Evaluation of NLCs based gel:}

\section{Viscosity}

Brookfield viscometer (Brookfield engineering laboratories, Inc., MA, USA) was used to determined the viscosity of the optimized NLCs gel formulation (Brook- 
field engineering laboratories, Inc., MA, USA) with spindle No. 62 at $10 \mathrm{rpm}$ at temperature of $37 \pm 0.5^{\circ} \mathrm{C}$.

\section{Determination of $\mathbf{p H}$}

Weighed quantity (1gm) of the NLCs preparation was taken and put in the 100 $\mathrm{mL}$ volumetric flask and made up the volume up to $50 \mathrm{ml}$ with distilled water (0.2\% strength). The $\mathrm{pH}$ was determined using $\mathrm{pH}$ meter ( $\mathrm{pH}$ Tutor Bench Meter, EUTECH Instruments, Singapore).

\section{Spreadability}

Glass plate was taken and marked a circle of $1 \mathrm{~cm}$ diameter. Then $0.5 \mathrm{~g}$ of NLCs gel was placed within a circle, over which another glass plate was placed. Half $\mathrm{kg}$ of weight was placed over the glass plate for 5-10 min. gel spreading diameter was noted and compare with the earlier one.

\section{Ex vivo permeation studies}

Ex vivo study was carried out using full thickness rat abdominal skin. In this work due to easy availability, the skin of albino rat was used. The species used was Wistar Albino Rats of 18-25 weeks and weight of 150-200 g of either sex. The abdominal skin was removed and dipped into phosphate buffer saline (PBS) pH 7.4. Hairs were removed from the skin by hair removal cream. The subcutaneous fat was removed with a scalpel. The skin was mounted on the Franz diffusion cell and the receptor chamber was filled with $20 \mathrm{ml}$ diffusion medium. The dispersion medium comprised of PBS pH 6.8 containing $0.8 \% \mathrm{v} / \mathrm{v}$ of Tween-80. The skin was situated on the receptor chamber with the stratum corneum facing upward in the receptor chamber and after that the donor chamber was clipped set up. The abundance skin was cut off and the entire get together was put on a magnetic stirrer to consistently mix the medium present in the receptor compartment. The distribution cell was set in the dispersion mechanical assembly to settle at $32^{\circ} \mathrm{C}$. The test formulations (Drug dispersion, Marketed formulation, optimized formulation, optimized gel formulation i.e., $1 \%$ cream) equivalent to $20 \mathrm{mg}$ drug were applied to the skin. Samples were withdrawn from the receptor compartment at predetermined time intervals, and immediately replaced with fresh diffusion medium. The studies were performed for $24 \mathrm{~h}$ according to the clinical application time and samples were analyzed spectrophotometrically at $\Lambda$ ${ }_{\max } 254 \mathrm{~nm}$. Drug permeation, Flux $\left(\mu \mathrm{g} / \mathrm{cm}^{2} / \mathrm{h}\right)$ and permeability coefficient $(\mathrm{Pb})$ $\left[\mathrm{cm}^{-2} \mathrm{~h}^{-1}\right]$ studies were calculated using the formulas mentioned ${ }^{29,30}$. 


\section{In vitro antifungal activity}

In vitro antifungal activity of the optimized formulation was determined by using cup plate method. In this method, firstly we prepared the dextrose agar media. Then sterilization was done by autoclaving and the sterilized media was transferred into the Petri plate for solidify ${ }^{31}$. After that inoculation of the fungal suspension was done in solidified media. The borer was used for the bore well must be Presterilized (equivalent to McFarland standard no. o.5). Presterilized stainless steel borer was used to bore wells in the media ${ }^{32}$. NLCs based gel; marketed formulation and BFZ STD solution were placed in respective bored well. Petri plates were allowed to incubate for growth of fungi and zone of inhibition was measured after incubation period ${ }^{33}$.

\section{In vivo Pharmacodynamics}

The in vivo Pharmacodynamics was performed according to the guidelines provided by institutional animal ethics committee (IAEC) (MMCP-IAEC-17).

According to the procedure required 12 male albino rats (weight 170-200gm) for Pharmacodynamic study. Proper diet and water was given for 24-48 h. Then the animals were divided into four different groups and each group contains three rats. Group 1 is control (untreated rats), groups 2, 3 and 4 consisted of rats induced with fungal infection and treated with Bifonazole loaded NLCs based Carbopol 940 gel, commercial Bifonazole cream (commercial formulation i.e., 1\% cream) and BFZ dispersion in water, respectively ${ }^{34}$. All sterilized materials were used.

\section{Preparation of fungal inoculums}

Candida albicans culture was obtained from Microbiology Department, MMIMS, Mullana, Ambala, India. The Candida albicans was subculture and allow for $24 \mathrm{hr}$ growing at $25^{\circ} \mathrm{C}$ and Adjusted the conidial suspension density to 1 $\times 10^{6} \mathrm{CFU} / \mathrm{ml}$ by hemocytometer and it was used for the inoculum ${ }^{35}$.

\section{Orientation of cutaneous candidiasis infection}

Firstly, $2 \mathrm{~cm}$ area on the back of the rat were make hairless by using hair removal cream and make a hairless square. On the same day, sand paper was used to abrade the skin. Ethyl alcohol was used to disinfect the skin and o.2ml conidial suspension was adhered to the skin by using sterile cotton and kept for 2-3 days ${ }^{35,36}$. Then the infected tissues of the skin was excite to the Sabouraud dextrose agar media with the help of sterile scalpel (at $25^{\circ} \mathrm{C}$ for 2 days) [36]. Appropriate dilution was done to accomplish countable colony forming unit (CFU) development. CFU was checked utilizing a computerized colony forming dependent on countable CFU values. This was preceded till acceptance of contagious 
disease was affirmed in view of the quantity of CFUs.

\section{Treatment}

BFZ loaded NLC based Carbopol 940 gel, BFZ dispersion and commercial formulation i.e., $2 \%$ cream (equivalent to $4 \mathrm{mg}$ drug on daily basis) were applied topically and the results in terms of reduction in fungal burden were compared. Treatment was started after confirmation of induction of fungal infection by counting the number of CFUs. After initiation of treatment, quantitative analysis of fungal burden was performed by the above mentioned procedure.

\section{RESULTS AND DISCUSSION}

\section{Selection of components}

The criteria for selection of excipients for developing BFZ-NLC include pharmaceutical acceptability, non-irritant and non-sensitizing to the skin and that they fall under GRAS (generally regarded as safe) category. As per the results of solubility studies, BFZ exhibited maximum solubility in Stearic acid, Castor oil and Tween 8o. Therefore, BFZ-NLC was prepared using Stearic acid as solid lipid, Castor oil as liquid lipid and Tween 80 as surfactant. Based on the visual observation of smear test, dual lipid phase was selected in the ratio 1:1 w/w (solid: liquid lipid ratio) for designing NLC.

\section{Particle size}

The particle size of the optimized NLCs was found to be in the nanometric size range (160.4 $\mathrm{nm}$ ) with low polydispersity index (0.338 \pm 0.16$)$ (figure 1.). it was observed the particle size decreased as the concentration of the liquid oily phase was increased. Furthermore. The increased concentration of the surfactant also influences the particle size of the Preparation. 


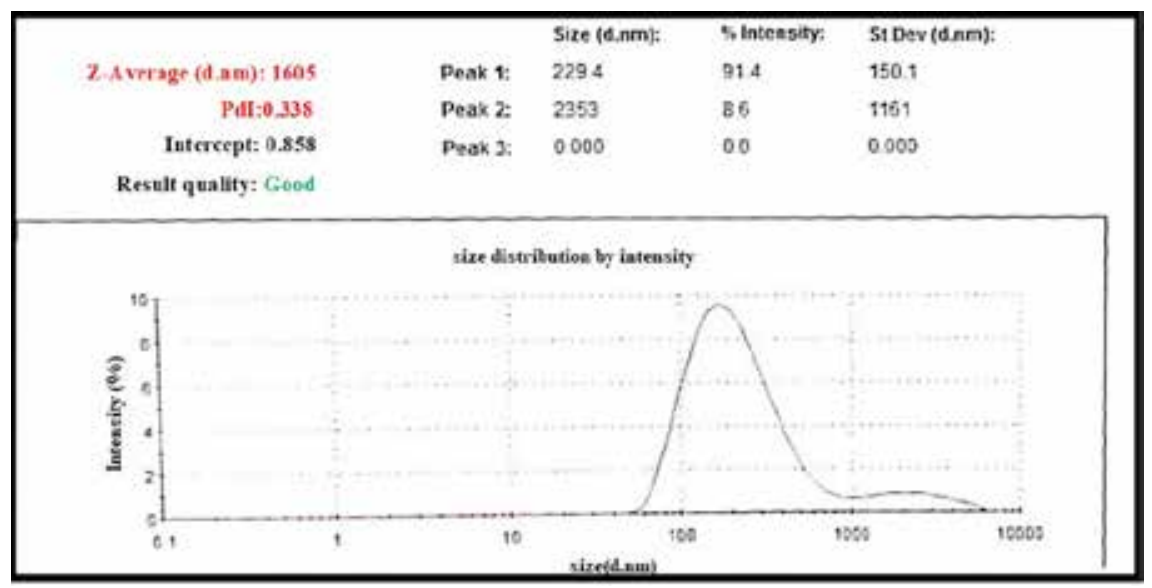

Figure 1: Particle size of optimized BFZ loaded NLCs formulation.

\section{Entrapment efficiency and drug loading}

The EE and DL was found to be $98.17 \pm .69$ and 19.6 \pm 1.23 . The EE and DL of the optimized BFZ-NLCs was Having the capability of lipid to integrate the drug and surfactant into the matrix. The solid lipid matrix encloses tiny oil section in which drug solubility is higher which increases their total drug loading capacity. And liquid lipid also affects the entrapment efficiency and helps in the loading of larger amount of drug (table 2).

\section{Optimization of BFZ loaded NLCs}

The $3^{2}$ factorial design was used to study the responses for all formulations on the basis of variables. The responses observed for all formulations were Particle size $\left(\mathrm{Y}_{1}\right)$, Entrapment Efficiency $\left(\mathrm{Y}_{2}\right)$ and Drug Loading $\left(\mathrm{Y}_{3}\right)$. Table 2 shows the experimental design of Stearic acid and castor oil nanoparticles and the results of measured responses. The effect of the combination of polymer and surfactant (Tween 80) on entrapment efficiency and drug loading was studied using the response surface plot and the results of the response surface plot are given in Figure $2(\mathrm{a}, \mathrm{b}, \mathrm{c})$. Based on the results obtained in preliminary experiments, the amount of Stearic acid and castor oil $\left(\mathrm{X}_{1}\right)$ and concentration of Surfactant $\left(\mathrm{X}_{2}\right)$ were found to be major variables affecting the Particle size $\left(\mathrm{Y}_{1}\right)$, Entrapment efficiency $\left(\mathrm{Y}_{2}\right)$ and \% Drug loading $\left(\mathrm{Y}_{3}\right)$ of the nanoparticles. In case of particle size, the results showed that an increase in PS due to an increase in the polymer concentration and a decrease in the volume of organic phase. In case of drug entrapment efficiency, the results indicate that an increase in drug entrapment due to an increase in polymer concentration and a decrease in the solvent. 


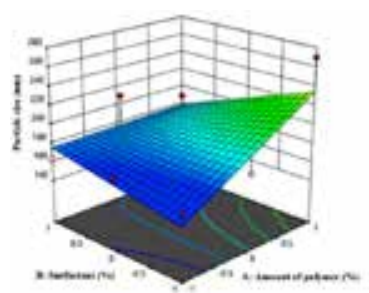

$\omega$

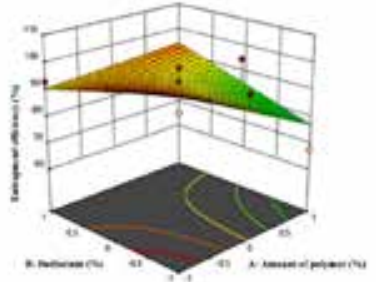

as

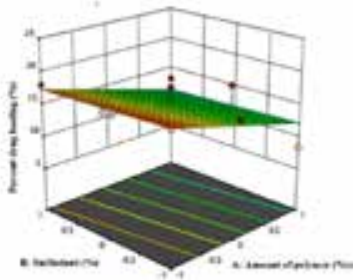

$(c)$

Figure 2: Response surface plots of factors (particle size, entrapment efficiency, drug loading

\section{Response surface plots}

Three-dimensional response surface plots generated by the Design Expert are presented in Fig 2(a) for bifonazole nanoparticles. Fig 2(a) depicts the response surface plots for the particle size of bifonazole nanoparticles which show an increase in PS due to an increase in the lipid concentration and a decrease in the volume of the aqueous phase $\mathrm{e}^{26,28}$. Fig 2(b) depicts the response surface plot for Drug entrapment efficiency which indicates an increase in drug entrapment due to an increase in lipid concentration. Fig 2(c) shows due to the concentration of lipids and surfactant the drug loading capacity was increased.

Quadratic model was found to be significant for particle size, entrapment efficiency and drug loading.

\section{Data Analysis}

The data generated by evaluation of the formulations were subjected to statistical analysis using $3^{2}$ full factorial designs with the help of design expert software version 9.0.5 (state-Ease, Inc., Minneapolis, USA). A statiscal model incorporating interactive and polynomial terms was used the evaluated the responses.

$\mathrm{Y}=\mathrm{b}_{\mathrm{o}}+\mathrm{b}_{1} \mathrm{X}_{1}+\mathrm{b}_{2} \mathrm{X}_{2}+\mathrm{b}_{12} \mathrm{X}_{1} \mathrm{X}_{2}+\mathrm{b}_{11} \mathrm{X}_{1}^{2}+\mathrm{b}_{22} \mathrm{X}_{2}^{2}$

Where $\mathrm{Y}$ is the independent variable. Where $b o$, the intercept is the arithmetic mean of the main effects (regression coefficients) b1, b2, b3, b12, b13, b23 and b123 were calculated by use of signs in the columns, by adding or subtracting the value of the obtained responses, $Y$. Finally, the values are summed up and divided with the number of formulations. Where $\mathrm{X}_{1}$ and $\mathrm{X}_{2}$ are the coded levels of the autonomous factors and $\mathrm{X}_{1} \mathrm{X}_{2}$ are the interaction and polynomial terms, respectively.

Based on the information acquired from the streamlined details, a general factual model can be depicted as for the above information. The model created can be 
described by utilizing the polynomial condition speaking to the separate reaction information. This can be given as takes after:

Particle Size $=184.70+22.17 \mathrm{X}_{1}-7.33 \mathrm{X}_{2}-20.50 \mathrm{X}_{1} \mathrm{X}_{2}$

Entrapment efficiency $=91.40-5164 \mathrm{X}_{1}+1.07 \mathrm{X}_{2}+7.53 \mathrm{X}_{1} \mathrm{X}_{2}$

Drug Loading $=14.83-3.01 \mathrm{X}_{1}-0.4383 \mathrm{X}_{2}$

From the above polynomial equations, 3D response surface graphs were generated, which were used to predict the responses of dependent variables at the intermediate levels of independent variables. Three dimensional response surface plots generated.

\section{Evaluation of NLCs}

\section{In vitro drug release study}

The in vitro drug release profiles (fig. 3) were used to determine \% cumulative drug release (CDR) varied widely b/w $56.38 \pm 2.26-92.5 \pm 1.18 \%$. This variability was observed due to variation in particle size among all the ten formulations.

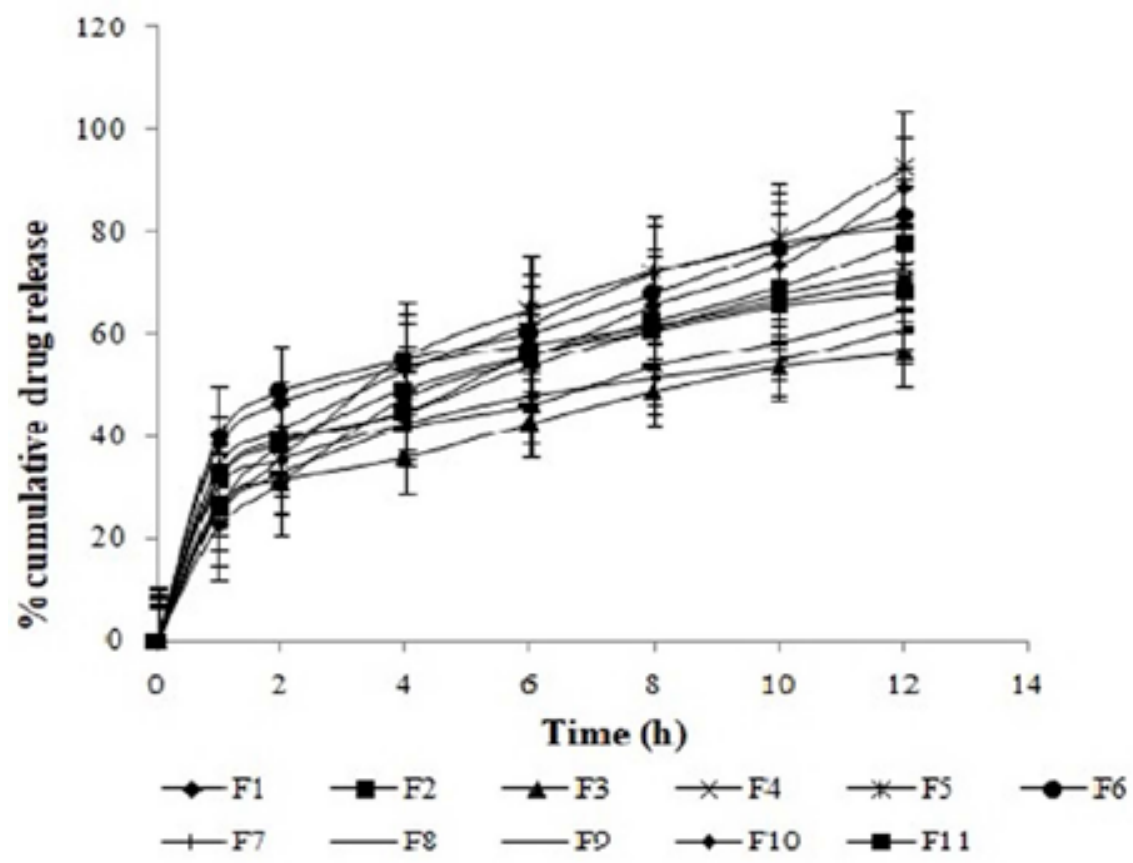

Figure 3: In vitro release of BFZ loaded NLCs formulations (F1-F11). 
Formulation F5, F6 \& F7 exhibited more than 80\% CDR and among these F5 displayed maximum CDR of $92.5 \pm 1.18 \%$ due to small sized particles and optimum entrapment efficiency NLCs developed from Span 80 (F1-F4) showed show \& incomplete CDR OF $(56.38 \pm 2.26-79.35 \pm 1.32)$ and when compared to F5 - F7 made with Tween 80 (81.2 $\pm 2.1-92.5 \pm 1.18)$. The determinants of this variability may be attributed to particle size and PDI that was in turn affected by type of copolymer used (Harada et al., 2011). Thus F8 - F11 prepared with PF127 produced large sized Nanostructured lipid carriers. This led to incomplete drug release which was varied from $(61.1 \pm 2.12-70.56 \pm 1.82 \%)$.

\section{Differential Scanning Calorimetry (DSC)}

The DSC thermogram shows the disappearance of drug peak in the optimized formulation ( $\mathrm{F}_{5}$ ) as shown in Fig 4. It means drug is fully enclosed inside the lyophilized drug-loaded NLCs.

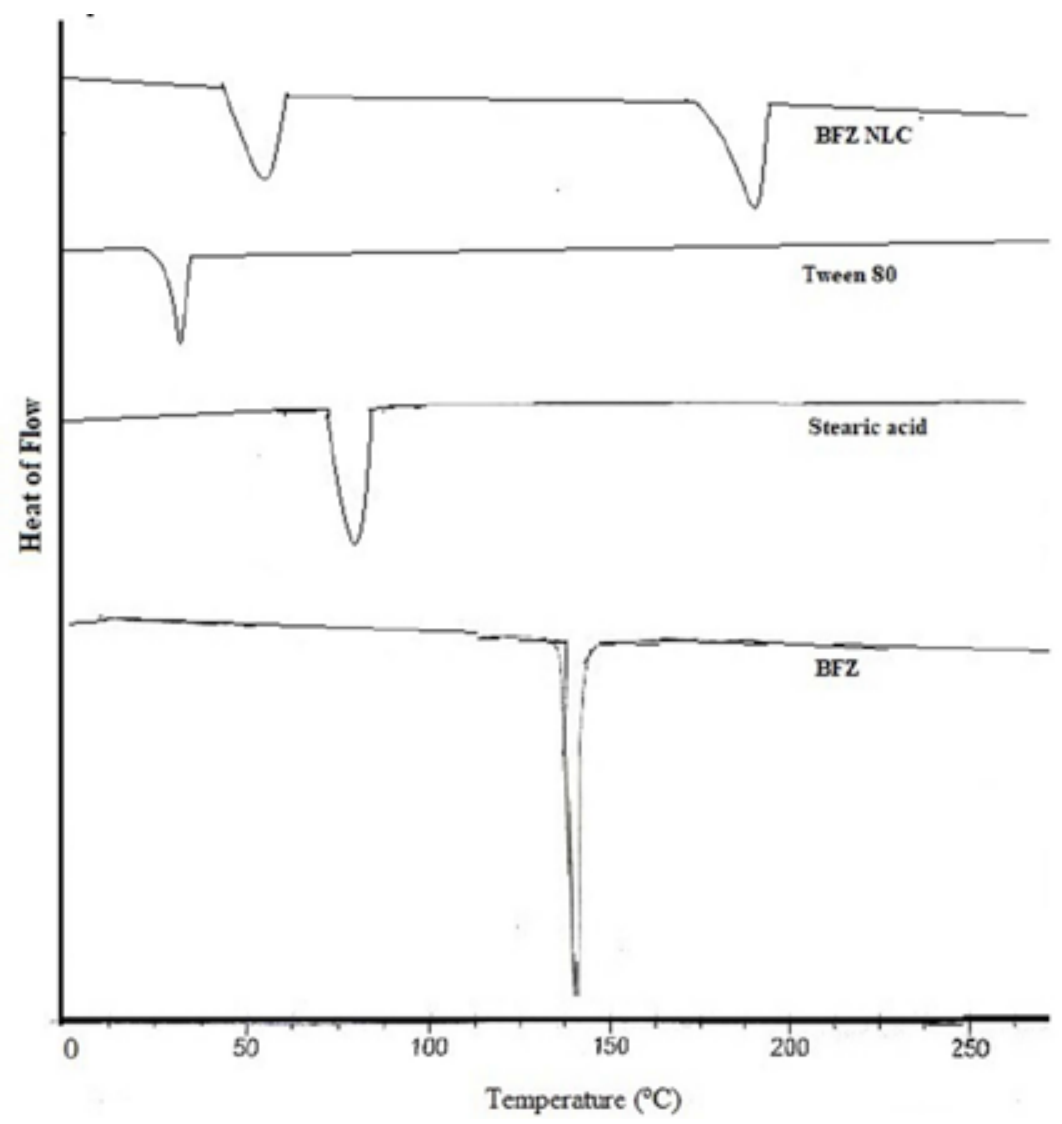

Figure 4: DSC curve of drug, lipids and surfactant. 


\section{Transmission electron microscopy (TEM)}

The TEM studies were carried out to get more insights into the morphology of the NLCs systems. From the study it was observed, that after loading the drug into placebo, the particle size of the formulation increased. This might be due to the accommodation of the drug in sufficient space in the lipid matrix. The TEM images (Fig 5) show the drug enclosed in the lipid matrix. The TEM images of BFZ-NLCs show uniform size distribution of lipid nanoparticles having coarsely spherical shape. The uniformity in particle size distribution correlates with the small PDI (o.38) obtained via photon correlation spectroscopy. The particle size after the TEM study was found to be in the range of $160-500 \mathrm{~nm}$.

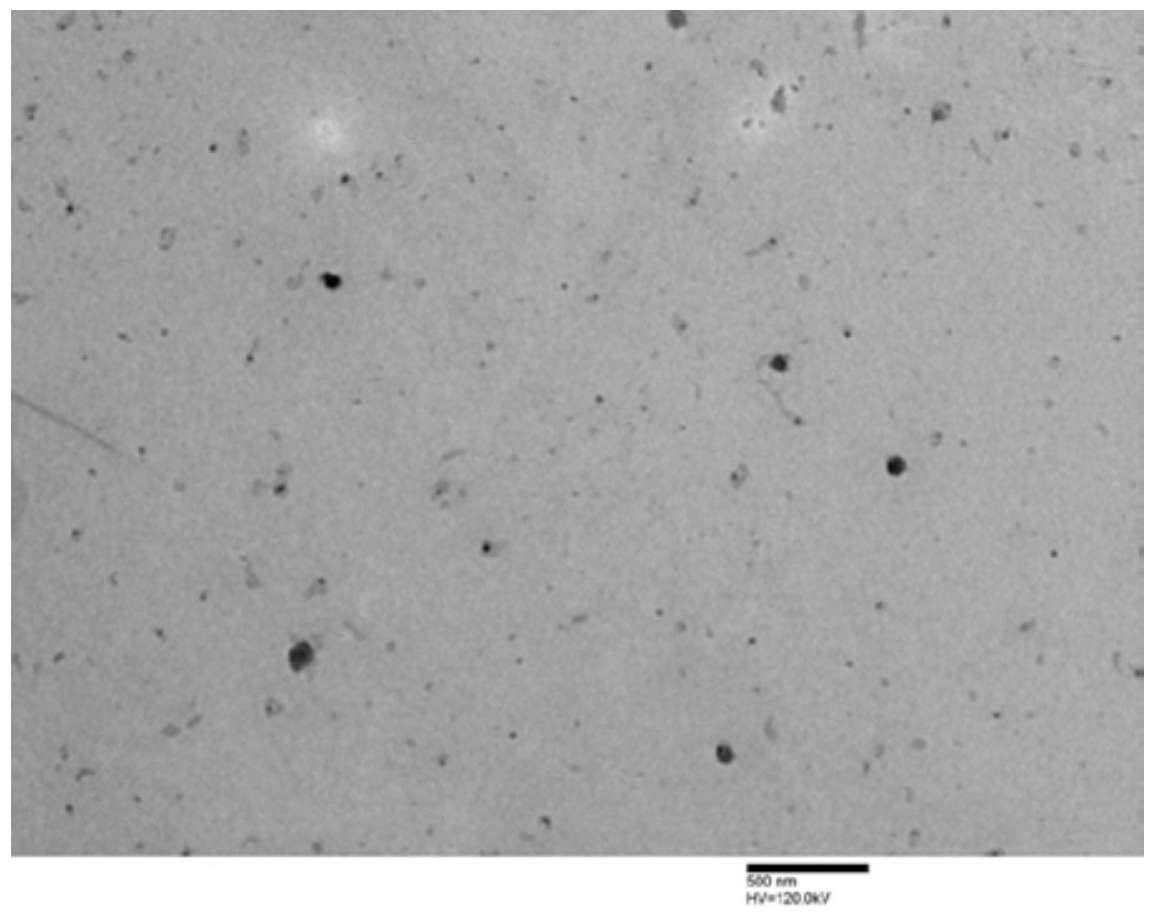

Figure 5: TEM image of optimized formulation. 


\section{Preparation of BFZ - NLCs based gel}

Carbopol 940 in various concentrations $1 \%, 1.5 \%$, and $2 \%$ was used to formulate the BFZ-NLCs into the gel. Gel (1.5\%) was found to be suitable for gelling the NLC because of desirable consistency.

\section{Viscosity}

Brookfield viscometer was used to determine the viscosity of the optimized NLCs gel formulation. The viscosity was found to be $593 \pm 0.98 \mathrm{cps}$.

\section{Determination of $\mathbf{p H}$}

$\mathrm{pH}$ Meter is used to determine the $\mathrm{pH}$ of the optimized NLCs Gel formulation in triplicate at $26^{\circ} \mathrm{C}$. and the $\mathrm{pH}$ was found to be $5.6 \pm 0.07$. The $\mathrm{pH}$ of the NLCloaded gel was within the acceptable range for topical formulations and compatible with the $\mathrm{pH}$ of the skin.

\section{Spreadability}

The ideal gelling formulation is readily spread on the site of application. The increased diameter due to the spreading of test gel formulation is found to be $6.5 \pm$ $0.05 \mathrm{~cm}$. the obtained value shows the good Spreadability of the test formulation and which is essential for the topical delivery

\section{Ex vivo permeation studies}

The aim if the recent study was determined the permeation of drug through the skin with controlled release effect. Drug loaded Nanostructured lipid carriers can easily penetrate the skin layers. Ex vivo permeation studies was performed for drug dispersion, marketed formulation, optimized formulation (F5) and gel formulation (G5) as shown in Fig 6. 


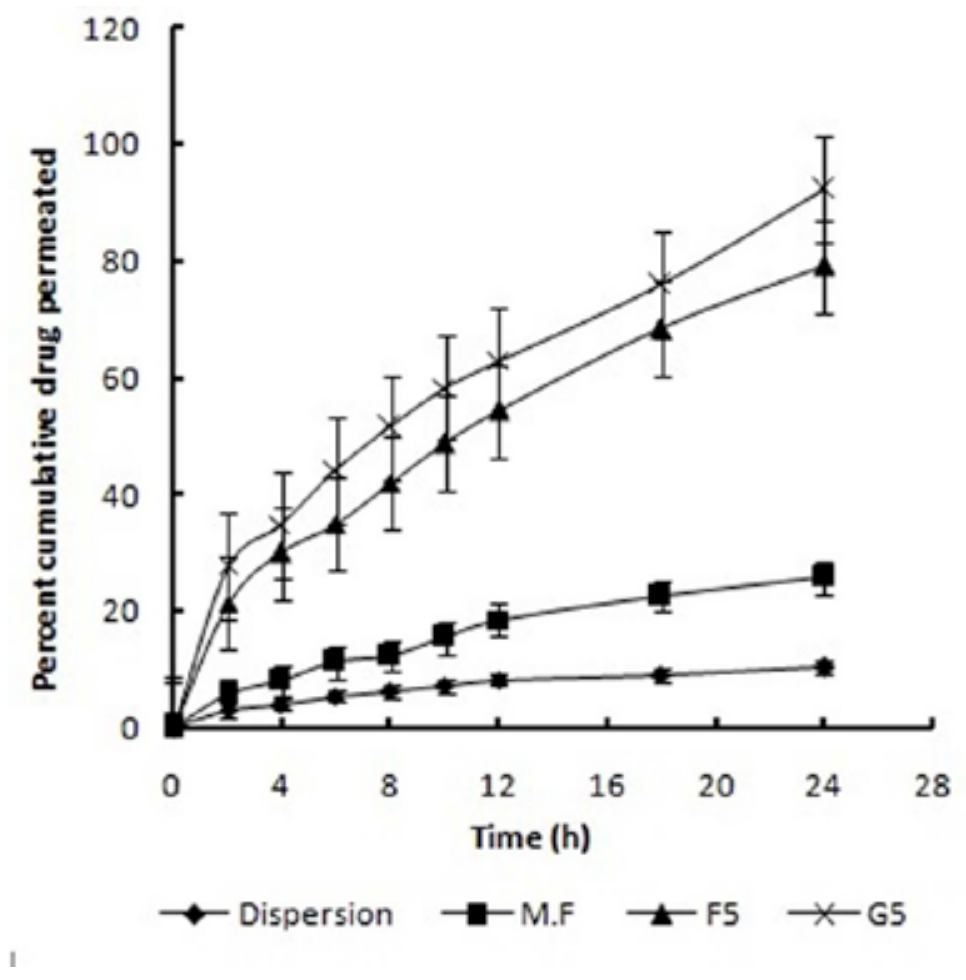

Figure 6: Comparative ex-vivo permeation of BFZ loaded optimized NLC (F5), Dispersion, M.F and G5.

The drug was dispersed within the lipid matrix which was further incorporated into Carbopol gel which adhered to the skin and increases the contact time. NLCs based gel and optimized formulation shows their skin targeting ability. This is desirable for the topical application. NLCs incorporated into gel may induce structural change of particle structure due to evaporation of water resulting in the transition of lipid matrix into a highly ordered structure causing drug expulsion $^{34}$. From this, it could be concluded that NLCs may play an important role in controlling the release of TH from NLCs as well as targeting of drug to the skin.

\section{In vitro antifungal activity}

The mean zone inhibition value of the NLCs gel was bigger than the marketed formulation but less than the BFZ standard solution.

NLCs based gel having the higher antifungal activity as compared to the market- 
ed formulation. This is due to the higher solubility of BFZ which helps to dipper penetration of BFZ loaded NLCs through the skin layer and inhibit the ergosterol synthesis $^{35}$ (Table 4).

\section{In vivo Pharmacodynamic}

Fungal burden was quantitatively analyzed in terms of colony forming units (CFUs) after initiation of the treatment. CFUs were counted using a colony counter (Microbiology lab, MMIMRS, Mullana, Ambala, India) (Table 5) gives the quantitative analysis of fungal burden.

Control group did not show any growth, as infection was not induced to this group. Group treated with NLCs showed a significant decrease in fungal burden after 5 days (671 \pm 40.675 CFUs) ( $p$ value < 0.001) as compared to CFUs before initiation of treatment $(2,55,000 \pm 3.505 .551 \mathrm{CFUs})$ ( $\mathrm{p}$ value $<0.001)$. Group treated with marketed formulation also showed a significant decrease in fungal burden after 5 days $(1674 \pm 154.65$ CFUs) ( $p$ value $<0.001)$, but it was higher as compared to the group treated with developed formulation. Also, the TH dispersion in water showed an initial reduction in fungal burden after which it almost came to a

Steady state ${ }^{36,37}$. These results showed that the NLC reduced the fungal burden in a shorter duration of time as compared to marketed formulation and dispersion. Thus, TH was found to be more effective when formulated as NLC based gel because of improved contact, adhesion, occlusion and sustained release.

BFZ loaded NLCs was successfully produced by high pressure homogenization technique using Stearic acid as solid lipid, Castor oil as liquid lipid and Tween 80 as surfactant. Therefore, BFZ loaded NLCs were capable of treating the fungal infection. And it can be concluded that the use of NLCs was far better than the conventional creams/gels. 


\section{TABLES}

Table 1: $3^{2}$ full factorial design layout for preparation of NLCs of BFZ

\begin{tabular}{lcccc}
\hline $\begin{array}{l}\text { Formulation } \\
\text { Code }\end{array}$ & $\begin{array}{c}\text { Bifonazole } \\
\text { (mg) }\end{array}$ & $\begin{array}{c}\text { Lipid ratio } \\
\text { (Castor oil: } \\
\text { Stearic acid) } \\
\text { (\% W/W) } \\
\mathbf{X}_{1}\end{array}$ & $\begin{array}{l}\text { Surfactant } \\
(\% \text { W/N) }\end{array}$ & $\begin{array}{c}\text { Dependent } \\
\text { variables }\end{array}$ \\
\hline F1 & 200 & $1: 1(-1)$ & $-1(1 \%)$ & \\
F2 & 200 & $1: 1(-1)$ & $+1(3 \%)$ & $\mathrm{Y}_{1=}$ Particle size \\
F3 & 200 & $4: 1(1)$ & $-1(1 \%)$ & $\mathrm{Y}_{2=} \%$ entrapment efficiency \\
\hline F4 & 200 & $1: 1(-1)$ & $0(2 \%)$ & $\mathrm{Y}_{3}=$ Drug loading \\
F5 & 200 & $4: 1(1)$ & $+1(3 \%)$ & \\
F6 & 200 & $2: 1(0)$ & $0(2 \%)$ & \\
F7 & 200 & $2: 1(-1)$ & $-1(1 \%)$ & \\
F8 & 200 & $4: 1(1)$ & $0(2 \%)$ & \\
F9 & 200 & $2: 1(0)$ & $+1(3 \%)$ & \\
${ }^{*}$ F10 & 200 & $1.5: 0.5$ & $(1.5 \%)$ & \\
${ }^{*}$ F11 & 200 & $2.5: 1.5$ & $(2.5 \%)$ & \\
\hline
\end{tabular}

Table 2: Results of $3^{2}$ factorial design of BFZ loaded nanoparticles

\begin{tabular}{|c|c|c|c|c|c|}
\hline $\begin{array}{c}\text { Formulation } \\
\text { Code. }\end{array}$ & $\begin{array}{c}\text { Lipid ratio } \\
\mathbf{( \% )} \\
\mathbf{X}\end{array}$ & $\begin{array}{c}\text { Surfactant } \\
\mathbf{( \% )} \\
\mathbf{X}_{\mathbf{1}}\end{array}$ & $\begin{array}{c}\text { Particle size } \\
\mathbf{( n m )} \\
\mathbf{Y}_{\mathbf{1}}\end{array}$ & $\begin{array}{c}\text { Entrapment } \\
\text { efficiency (\%) } \\
\mathbf{Y}_{2}\end{array}$ & $\begin{array}{c}\text { Drug loading } \\
\mathbf{( \% )} \\
\mathbf{Y}_{3}\end{array}$ \\
\hline F1 & $1: 1(-1)$ & $1(-1)$ & 162.50 & $98.02 \pm 1.00$ & $18.70 \pm 1.25$ \\
\hline F2 & $1: 1(-1)$ & $3(+1)$ & 174.20 & $96.30 \pm 1.50$ & $16.50 \pm 0.58$ \\
\hline F3 & $4: 1(+1)$ & $1(-1)$ & 270.62 & $67.60 \pm 1.23$ & $08.38 \pm 2.36$ \\
\hline F4 & $1: 1(-1)$ & $2(0)$ & 169.20 & $97.78 \pm 1.12$ & $17.70 \pm 3.20$ \\
\hline F5 & $4: 1(+1)$ & $3(+1)$ & 160.40 & $98.17 \pm 0.69$ & $19.6 \pm 1.23$ \\
\hline F6 & $2: 1(0)$ & $2(0)$ & 168.30 & $94.65 \pm 0.98$ & $15.04 \pm 2.14$ \\
\hline F7 & $2: 1(0)$ & $1(-1)$ & 163.40 & $93.06 \pm 1.02$ & $18.90 \pm 1.23$ \\
\hline F8 & $4: 1(+1)$ & $2(0)$ & 210.30 & $82.54 \pm 1.62$ & $10.07 \pm 2.75$ \\
\hline F9 & $2: 1(0)$ & $3(-1)$ & 189.60 & $92.76 \pm 1.71$ & $11.75 \pm 3.12$ \\
\hline *F10 & $1.5: 0.5$ & 1.5 & 182.70 & $93.16 \pm 0.98$ & $14.67 \pm 1.29$ \\
\hline *F11 & $2.5: 1.5$ & 2.5 & 184.60 & $94.31 \pm 0.56$ & $15.45 \pm 1.87$ \\
\hline
\end{tabular}


Table 3: In vitro release profile of BFZ loaded NLCs

\begin{tabular}{|c|c|c|c|c|c|c|c|c|c|cc|}
\hline \multirow{2}{*}{$\begin{array}{c}\text { Time } \\
\text { (h) }\end{array}$} & \multicolumn{10}{|c|}{ Formulation code } \\
\cline { 2 - 13 } & F1 & F2 & F3 & F4 & F5 & F6 & F7 & F8 & F9 & F10 & F11 \\
\hline $\mathbf{0}$ & $\mathbf{0}$ & $\mathbf{0}$ & $\mathbf{0}$ & $\mathbf{0}$ & $\mathbf{0}$ & $\mathbf{0}$ & $\mathbf{0}$ & $\mathbf{0}$ & $\mathbf{0}$ & $\mathbf{0}$ & $\mathbf{0}$ \\
\hline $\mathbf{1}$ & 22.31 & 26.45 & 26.90 & 31.70 & 25.53 & 40.40 & 34.60 & 30.40 & 25.30 & 38.34 & 32.34 \\
\hline $\mathbf{2}$ & 30.74 & 38.22 & 31.40 & 38.30 & 35.45 & 48.50 & 41.30 & 35.40 & 32.70 & 46.43 & 39.90 \\
\hline $\mathbf{4}$ & 47.56 & 49.30 & 35.61 & 44.60 & 55.43 & 54.94 & 52.78 & 42.56 & 41.60 & 53.40 & 44.60 \\
\hline $\mathbf{6}$ & 55.34 & 55.60 & 42.32 & 53.76 & 64.65 & 60.23 & 61.90 & 47.60 & 45.90 & 57.90 & 55.90 \\
\hline $\mathbf{8}$ & 65.40 & 62.34 & 48.80 & 61.20 & 72.30 & 67.80 & 71.68 & 51.30 & 53.70 & 61.60 & 60.80 \\
\hline $\mathbf{1 0}$ & 73.42 & 69.21 & 53.60 & 67.56 & 78.6 & 76.54 & 77.90 & 54.80 & 58.34 & 66.40 & 65.30 \\
\hline $\mathbf{1 2}$ & 79.35 & 78.12 & 56.38 & 72.62 & 92.50 & 83.40 & 81.20 & 61.10 & 64.80 & 70.56 & 68.34 \\
\hline
\end{tabular}

Table 4: Comparison of NLCs based gel, marketed formulation and Standard drug for antifungal activity

\begin{tabular}{c|cc|}
\hline S. no & Formulation & $\begin{array}{c}\text { Mean zone of inhibition in } \mathbf{c m}(\mathbf{n}=\mathbf{3}) \\
\text { (Mean } \pm \text { SD) }\end{array}$ \\
\hline 1 & BFZ Standard & $1.45 \pm 0.12$ \\
\hline 2 & NLCs based gel & $1.29 \pm 0.09$ \\
\hline 3 & Marketed formulation & $1.15 \pm 0.87$ \\
\hline
\end{tabular}

\section{ACKNOWLEDGEMENT}

The authors are thankful to M. M. College of Pharmacy, Maharishi Markandeshwar (Deemed to be University), Mullana, Ambala, Haryana for providing the facilities and instruments to carry out this work.

\section{COMPLIANCE WITH ETHICAL STANDARDS}

For this study, prior clearance from an institutional animal ethics committee (approval number MMCP-IAEC-17) was obtained.

\section{CONFLICT OF INTEREST}

The authors declare that there is no conflict of interest. The authors alone are responsible for content and writing of the paper.

\section{DISCLAIMER}

The authors alone are responsible for the content and writing of the paper. 


\section{REFERENCES}

1. Warnock, D.W. Trends in the epidemiology of invasive fungal infections. Jpn. J. Med. Mycol. $\mathbf{2 0 0 7 , ~ 4 8 , ~ 1 - 1 2 . ~}$

2. Bangia, R.; Sharma, G.; Dogra, S.; Katare, O.P. Nanotechnological interventions in dermatophytosis: from oral to topical, a fresh perspective. Expert Opinion on Drug Delivery. 2o19, $16,377-396$.

3. Wishart, D.S.; Knox, C.; Guo, A.C.; Cheng, D.; Shrivastava, S. Drug Bank: a knowledgebase for drugs, drug actions and drug targets. Nucleic Acids Res. 2008, 36, D901-D9o6.

4. Müller-Goymann, C.C. Physiochemical characterization of colloidal drug delivery systems such as reverse micelles, vesicles, liquid crystals and nanoparticles for topical administration. Eur. J. Pharm. Biopharm. 2004, 58, 343-356.

5. O'Driscoll, C.M.; Griffin, B.T. Biopharmaceutical challenges associated with drugs with low aqueous solubility - The potential impact of lipid based formulations. Adv. Drug Deliv. Rev. 2008, 60, 617-24.

6. Moazeni, M.; Saeedi, M.; Kelidari, H.; Nabili, M.; Davari, A. An update on the application of nano-scaled carriers against fluconazole-resistant Candida species: nanostructured lipid carriers or solid lipid nanoparticles. Curr. Med. Mycol. 2019, 5, 8-13.

7. Gaba, B.; Fazil, M.; Khan, S.; Ali, A.; Ali, J. The next generation after the liposomes: solid lipid NPs (SLNTM) as dermal carrier in cosmetics. Bull. Fac. Pharm. Cairo Univ. 2015, 53(2), 147-159.

8. Muller, R.; Mader, K.; Gohla, S. Solid lipid nanoparticles (SLN) for controlled drug delivery A review of the state of the art. Eur. J. Pharm. Biopharm. 2ooo, 50, 161-77.

9. Mehnert, W.; Mader, K. Solid lipid NPs: production, characterization and applications. Adv Drug Deliv Rev 2oo1, 47, 165-96.

10. Souto, E.B.; Wissing, S.A.; Barbosa, C.M.; Muller, R.H. Development of a controlled release formulation based on SLN and NLC for topical clotrimazole delivery. Int. J. Pharm. 2004, 271(1), 71-7.

11. Puri, A.; Loomis, K.; Smith, B.; Lee, J.H.; Yavlovich, A.; Heldman, E. R. Lipid-Based Nanoparticles as Pharmaceutical Drug Carriers: From Concepts to Clinic. Crit Rev Ther Drug Carrier Syst. 2009, 26(6), 523-580.

12. Qiuyu, Wei; Qiuxuan, Yang; Qilong; Wang; Congyong, Sun; Yuan, Zhu; Ya, Niu;Jiangnan, Yu; Ximing, Xu. Formulation, Characterization, and Pharmacokinetic Studies of 6-Gingerol-Loaded Nanostructured Lipid Carriers. AAPS PharmSciTech. 2018, 19(8), 3661-3669.

13. Upendra Nagaich, U.; Gulat, N. Nanostructured lipid carriers (NLC) based controlled release topical gel of clobetasol propionate: design and in vivo characterization. Drug Deliv. Transl. Res. 2016, 6(3), 289-98.

14. Gaba, B.; Fazil, M.; Ali, A.; Baboota, S.; Sahni, J.K.; Ali, J. Nanostructured lipid (NLCs) carriers as a bioavailability enhancement tool for oral administration. Drug Deliv. 2015; 22 (6):691-700.

15. Alberti, I.; Kalia, Y.N.; Naik, A.; Bonny, J.; Guy, R.H. Effect of ethanol and isopropyl myristate on the availability of topical terbinafine in human stratum corneum, in vivo. Int. J. Pharm. 2001, 219, 11-9.

16. Tapeinos, C.; Battaglini, M.; Ciofani, G. Advances in the design of solid lipid nanoparticles and nanostructured lipid carriers for targeting brain diseases. J Control Release. 2017, 264, 306-332. 
17. Claudia, V.; Katharina, S. Influence of carrageenan on the rheology and skin permeation of microemulsion formulations. J Control release 2004, 95(2), 257-65.

18. Pople, P.V.; Singh, K.K. Development and evaluation of colloidal modified nanolipid carrier: Application to topical delivery of tacrolimus. Eur. J. Pharm. Biopharm. 2011, 79, 82-94.

19. Khalil, R.M.; Abd- Elbary, A.; Kassem, M.A.; El Ridy, M.S.; Samra, G.E.A.; Awad, G.E.A. et al. Formulation and characterization of nystatin loaded nanostructured lipid carriers for topical delivery against cutaneous candidiasis. Br. J. Pharm. Res. 2014, 4(4), 490-512.

20. Patel, D.; Dasgupta, S.; Dey, S.; Ramani, Y.R.; Ray, S.; Mazumder, B. Nanostructured lipid carriers (NLC)-based gel for the topical delivery of aceclofenac: preparation, characterization, and in vivo evaluation. Sci. Pharm. 2012, 80, 749-64.

21. Bhalekar, M.R.; Pokharkar, V.; Madgulkar, A.; Patil, N. Preparation and evaluation of miconazole nitrate-loaded solid lipid nanoparticles for topical delivery. AAPS Pharm. Sci. Tech. 2009, 10(1), 289-96.

22. Shete, H.; Patravale, V.S. Long chain lipid based tamoxifen NLC. Part I: Preformulation, formulation development and physicochemical characterization. Int. J. Pharm. 2013, 454(1), $573-83$.

23. Bali, V.; Ali, M.; Ali, J. Study of surfactant combinations and development of a novel nanoemulsion for minimising variations in bioavailability of ezetimibe. Colloids Surf B Biointerf. 2010, 76, 410-20.

24. Gaba, B.; Fazil, M. Nanostructured lipid carrier system for topical delivery of terbinafine hydrochloride. Bull. Fac. Pharm. Cairo Univ. 2015, 53, 147-159.

25. Muller, R.; Mader, K.; Gohla, S. Solid lipid nanoparticles (SLN) for controlled drug delivery - A review of the state of the art. Eur. J. Pharm. Biopharm. 2000, 50, 161-77.

26. Baboota, S.; Al-Azaki, A.; Kohli, K.; Ali, J.; Dixit, N.; Shakeel, F. Development and evaluation of a microemulsion formulation for transdermal delivery of terbinafine. PDA J Pharm. Sci. Technol. 2007, 61(4), 276-85.

27. Sanad, R.A.; Abdel Malak, N.S.; Bayoomy, T.S.; Badawi, A.A. Formulation of novel oxybenzone-loaded nanostructured lipid carriers (NLCs). AAPS Pharm. Sci. Tech. 2010, 11(4), 1684-94.

28. Fang, J.Y.; Fang, C.L.; Liu, C.H.; Su, Y.H. Lipid nanoparticles as vehicles for topical psoralen delivery: solid lipid nanoparticles (SLN) versus nanostructured lipid carriers (NLC). Eur. J. Pharm. Biopharm. 2008, 70, 633-40.

29. Cirri, M.; Bragagni, M.; Menni, N.; Mura P. Development of a new delivery system consisting in "drug - in cyclodextrin - in nanostructured lipid carriers" for ketoprofen topical delivery. Eur. J. Pharm. Biopharm. 2012, 80(1), 46-53.

30. Ananthanarayan, R.; Panicker, C.K.J. Laboratory Control of Antimicrobial Therapy. Textbook of Microbiology, India, Universities Press. 2009, 246-247.

31. Sabale, V.; Vora, S. Formulation and evaluation of microemulsion-based hydrogel for topical delivery. Int. J. Pharm. Investig. 2012, 2, 140-149.

32. Pottoo, F.H.; Sharma, S; Javed, M.N.; Barkat, M..A; Harshita, Alam, M.S; Naim, M.J.; Alam, M.O; Ansari, M.A,; Barreto, G.E; Ashraf, G.M. Lipid-based nanoformulations in the treatment of neurological disorders. Drug Metab. Rev. 2020, 2, 1-20.

33. Bachhav, Y.G.; Patravale, V.B. Microemulsions-based vaginal gel of clotrimazole: formulation, in vitro evaluation and stability studies. AAPS Pharm. Sci.Tech. 2009, 10(2), 476-481.

34. Patel, M.R.; Patel, R.B.; Parikh, J.R.; Bhatt, K.K. Investigating effect of microemulsion com- 
ponents: In vitro permeation of ketoconazole. Pharm. Dev. Technol. 2011, 16(3), 250-258.

35. Uchida, K.; Tanaka, T.; Yamaguchi, H. Achievement of complete mycological cure by topical antifungal agent NND-502 in guinea pig model of tinea pedis. Microbiol Immunol. 2003, 47, $143-6$.

36. Tatsumi, Y.; Yokoo, M.; Arika, T.; Yamaguchi, H. In vitro antifungal activity of KP-103, a novel triazole derivative, and its therapeutic efficacy against experimental plantar tinea pedis and cutaneous candidiasis in guinea pigs. Antimicrob Agents Chemother. 2001, 45, 1493-9.

37. Khosa, A.; Reddi, S.; Saha, R.N. Nanostructured lipid carriers for site-specific drug delivery. Biomed. Pharmacother. 2018, 103, 598-613. 